\title{
Alternativas de retenção para reabilitação bucal com prótese obturadora maxilar após maxilectomia parcial
}

\section{Retention alternatives for mouth rehabilitation with maxillary obturator prosthesis after partial} maxilectomy

Alternativas de retención para la rehabilitación bucal con prótesis obturadora maxilar después de la maxilectomía parcial

Fernanda Cristina Cunha da Silva ORCID: https://orcid.org/0000-0001-5067-3578 Universidade do Estado do Amazonas, Brasil E-mail: fccs.odo17@uea.edu.br

Cristiane Maria Brasil Leal

ORCID: https://orcid.org/0000-0002-7826-0174 Universidade do Estado do Amazonas, Brasil E-mail: cbleal@uea.edu.br

Guilherme Barbosa de Freitas

ORCID: https://orcid.org/0000-0002-2284-1068 Universidade do Estado do Amazonas, Brasil E-mail: gbf.odo17@uea.edu.br

Lara Pepita de Souza Oliveira

ORCID: https://orcid.org/0000-0002-5667-0877

Universidade Federal do Amazonas, Brasil E-mail: larapepita1@gmail.com

Sybilla Torres Dias

ORCID: https://orcid.org/0000-0003-1838-9598 Universidade do Estado do Amazonas, Brasil E-mail: sdias@uea.edu.br

Brigitte Nichthauser

ORCID: https://orcid.org/0000-0003-3736-8856 Universidade do Estado do Amazonas, Brasil E-mail: brigitte@uea.edu.br

Francisco Pantoja Braga

ORCID: https://orcid.org/0000-0001-6119-6973

Universidade do Estado do Amazonas, Brasil E-mail: fbraga@uea.edu.br

\begin{abstract}
Resumo
Ressecções cirúrgicas de tumores maxilares podem causar comunicação bucosinusal que possuem como método de reabilitação a confecção de prótese obturadora maxilar. Sendo os aspectos biomecânicos o principal desafio para um tratamento protético eficaz. Este artigo visa relatar um caso de reabilitação com prótese obturadora maxilar após maxilectomia parcial. Paciente do gênero feminino, 14 anos, procurou atendimento odontológico desejando substituir a prótese obturadora provisória que havia sido confeccionada três anos atrás, para correção de defeito maxilar gerado pela remoção cirúrgica de um osteoma. Após anamnese, exames intra e extra-oral optou-se pela realização de uma prótese parcial removível superior com obturador palatino. Iniciou-se pela moldagem das duas arcadas para obtenção dos modelos de estudo onde foi realizado o delineamento e planejamento do caso. Após realização do preparo de boca e moldagem funcional, obteve-se o modelo de trabalho, utilizado para confecção da armação metálica que foi provada e ajustada na boca analisando os aspectos biomecânicos. Após registro do arco facial e determinação das relações intermaxilares os modelos foram montados em articulador semi-ajustável, executada a seleção e montagem dos dentes artificiais. Em seguida foi realizada a prova estética e funcional e ajustes necessários. Posteriormente a moldagem de boca fechada, foram realizadas a acrilização, polimerização, instalação e proservações da prótese. A prótese obturadora se mostrou eficaz, em seu aspecto biomecânico, gerando boa retenção e estabilidade. Sendo a prótese parcial removível uma alternativa favorável a reabilitação de pacientes submetidos a cirurgias mutiladoras maxilofaciais.
\end{abstract}

Palavras-chave: Obturadores palatinos; Prótese maxilofacial; Reabilitação bucal; Retenção da prótese; Osteoma. 


\begin{abstract}
Surgical resections of maxillary tumors may cause bucosinusal communication that have as a rehabilitation method the manufacture of maxillary obturator prosthesis. Biomechanical aspects are the main challenge for effective prosthetic treatment. This article aims to report a rehabilitation case with maxillary obturator prosthesis after partial maxilectomy. A 14-year-old female patient sought dental care desiring to replace the provisional obturator prosthesis that had been made three years ago, to correct the maxillary defect generated by the surgical removal of an osteoma. After anamnesis, intra- and extra-oral examinations were chosen to perform a removable partial upper prosthesis with a palatine shutter. It was started by molding the two arcades to obtain the study models where the design and planning of the case was carried out. After mouth preparation and functional molding, the work model was obtained, used to make a metal frame that was proven and adjusted in the mouth analyzing the biomechanical aspects. After recording the facial arch and determining the intermaxillary relationships, the models were mounted on a semi-adjustable articulator, performed the selection and assembly of artificial teeth. Then, the aesthetic and functional test and necessary adjustments were performed. Subsequently, the molding of the closed mouth was performed, acrylization, polymerization, installation and proservations of the prosthesis were performed. The obturator prosthesis proved to be effective, in its biomechanical aspect, generating good retention and stability. The removable partial prosthesis is a favorable alternative to rehabilitation of patients undergoing maxillofacial mutilator surgeries.
\end{abstract}

Keywords: Palatal obturators; Maxillofacial prosthesis; Mouth rehabilitation; Prosthesis retention; Osteoma.

\title{
Resumen
}

Las resecciones quirúrgicas de tumores maxilares pueden causar comunicación bucosinusal que tienen como método de rehabilitación la fabricación de prótesis obturadoras maxilares. Los aspectos biomecánicos son el principal desafío para un tratamiento protésico eficaz. Este artículo tiene como objetivo reportar un caso de rehabilitación con prótesis obturadora maxilar después de maxilectomía parcial. Paciente femenina, 14 años, buscó atención dental deseando reemplazar la prótesis obturadora provisional que le habían realizado hace tres años, para corregir un defecto maxilar causado por la extirpación quirúrgica de un osteoma. Después de la anamnesis, exámenes intra y extraorales, se decide realizar prótesis parcial superior removible con obturador palatino. Empiezo por moldear las ds arcadas para obtener los modelos de estudio donde se realizó el diseño y planificación del caso. Después de la preparación de la boca y el moldeo funcional, se obtuvo el modelo de trabajo, utilizado para hacer un marco metálico que fue probado y ajustado en la boca analizando los aspectos biomecánicos. Después de registrar el arco facial y determinar las relaciones intermaxilares, los modelos fueron montados en un articulador semiajustable, se realizó la selección y montaje de dientes artificiales. Luego, se realizó la prueba estética y funcional y los ajustes necesarios. Luego del moldeo a boca cerrada, se realizó la acrilización, polimerización, colocación y conservación de la prótesis. La prótesis obturadora demostró ser eficaz, en su aspecto biomecánico, generando buena retención y estabilidad. La prótesis parcial removible es una alternativa favorable a la rehabilitación de pacientes sometidos a cirugías mutilación maxilofacial.

Palabras clave: Obturadores palatinos; Prótesis maxilofacial; Rehabilitación bucal; Retención de la prótesis; Osteoma.

\section{Introdução}

Os osteomas são tumores ósseos benignos compostos de osso maduro compacto ou medular, apresentam crescimento lento, progressivo e indolor. Em sua maioria, são diagnosticados durante a adolescência, por exames radiográficos de rotina. A lesão é exclusiva aos ossos craniofaciais, e as localizações anatômicas preferenciais são maxila, mandíbula e os seios paranasais, em que o seio frontal é o mais frequente. O tratamento preferencial é a remoção cirúrgica do tumor, sendo a recidiva da lesão extremamente rara (Neville et al., 2016; Boros et al., 2011).

A intervenção cirúrgica na maxila é a maxilectomia parcial ou total, sendo a abordagem reparadora obtida por meio de retalhos locais, transferências de tecido livre, enxertos não vascularizados e obturadores protéticos. Dessas alternativas apenas os retalhos cirúrgicos surgem como correção definitiva para comunicação bucosinusal (Goiato et al., 2009; Singh et al., 2013).

Os obturadores protéticos visam separar a cavidade oral e nasal, restabelecendo o equilíbrio funcional do aparelho estomatognático, facilitando assim a deglutição, mastigação, fonação, reduzindo os distúrbios da articulação temporomandibular (Singh et al., 2013). Além disso, promove a visualização da área operada, contribuindo para o controle de possíveis recidivas da lesão. A prótese obturadora oferece uma reabilitação em curto período. (Dings et al., 2018; Singh et al., 2013).

Os defeitos maxilares podem ser definidos de acordo com a classificação de Aramany (1978), que relaciona a 
ressecção maxilar e os dentes remanescentes com o grau de retenção e estabilidade dos obturadores protéticos. A retenção é afetada por diversos fatores, entre eles o número de dentes remanescentes, o tamanho do defeito maxilar após a maxilectomia, o controle muscular, o tecido disponível ao redor da cavidade e a massa presente na estrutura protética (Aramany, 1978; Ali et al., 2018).

Os dentes restantes interferem diretamente na retenção das próteses parciais removíveis com obturadores palatinos, pois o número, distribuição, condição periodontal e a anatomia influenciam nos deslocamentos protéticos. Outras formas de se obter a retenção e estabilidade protética é por meio de implantes osseointegrados, como os encaixes do tipo O'ring, barra clipe, sistema ERA, além da utilização dos magnetos (Singh et al., 2013; Kusterer et al., 2012).

Torna-se importante o entendimento acerca dos benefícios funcionais e biomecânicos da reabilitação bucal alcançada por meio das próteses obturadoras. Por isto, o objetivo deste artigo é relatar um caso clínico de reabilitação com prótese parcial removível superior com obturador palatino, após maxilectomia parcial para remoção de osteoma, que resultou em uma comunicação bucosinusal.

\section{Metodologia}

Este artigo retrata um relato de caso clínico, de abordagem qualitativa e caráter descritivo (Pereira et al., 2018), descreve uma das possibilidades utilizada como tratamento reabilitador bucal para pacientes com defeitos maxilares de etiologia adquirida, encontrados em ressecções cirúrgicas de tumores. Evidencia a importância da prótese parcial removível como método retentivo para os obturadores palatinos. $\mathrm{O}$ artigo preserva a identidade da paciente, sendo realizado mediante assinatura do Termo de Consentimento Livre e Esclarecido- TCLE.

\section{Relato de Caso}

Paciente AMC, gênero feminino, melanoderma, 14 anos, compareceu ao Centro de Especialidades Odontológicas da Universidade do Estado do Amazonas, CEO/UEA, para confecção de uma prótese obturadora palatina pois a prótese em uso apresentava adaptação e função comprometida devido ao crescimento fisiológico da paciente.

$\mathrm{Na}$ anamnese a paciente relatou que a três anos atrás havia sido submetida a tratamento cirúrgico de maxilectomia parcial para excisão de um osteoma e que em seguida foi instalada uma prótese obturadora palatina provisória que precisava ser trocada. Foi verificado que a paciente apresentava boa saúde geral, com ausência de doenças sistêmicas. Ao exame clínico extraoral observou-se assimetria facial, perda de continuidade da pele do lado esquerdo, inexistência de sintomatologia dolorosa e edema (Figura 1).

Figura 1: Aspecto extraoral inicial. A) Frontal. B) Perfil direito. C) Perfil esquerdo.
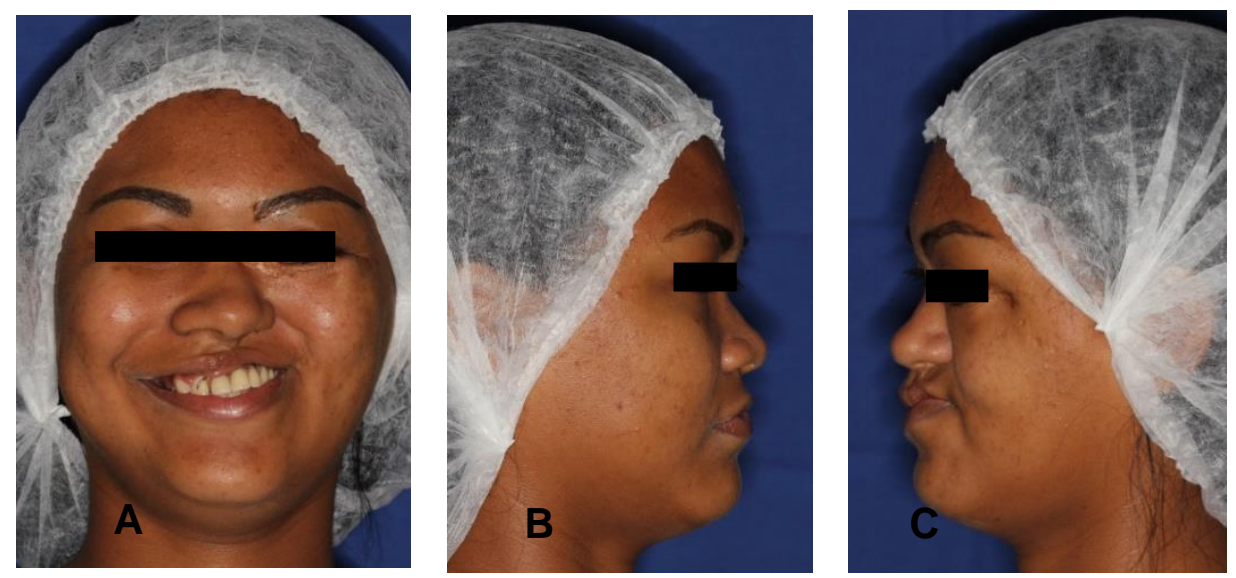

Fonte: Autores. 
A Figura 1 mostra a aparência extra-oral inicial da paciente podendo-se verificar a assimetria entre o lado esquerdo e direto da face, tanto na vista frontal como na comparação ente o perfil direito e o esquerdo.

No exame clínico intraoral foi verificado a ausência dos elementos dentários 11, 21-27, comunicação bucosinusal, inexistência de cárie e lesão periodontal (Figura 2). O estado clínico da paciente induziu prejuízos estéticos e funcionais, relacionados a fonação, mastigação e deglutição. Na arcada inferior, não havia ausência de elementos dentários. Dessa forma, considerando que todos os elementos dentários já se encontravam erupcionados na cavidade bucal, optou-se pela confecção de uma prótese parcial removível superior com obturador palatino. O tratamento foi bem aceito pela paciente e sua mãe e o Termo de Consentimento Livre e Esclarecido (TCLE) foi assinado.

Figura 2: Aspecto inicial da cavidade bucal. A) Comunicação Bucosinusal. B) Ausência de rebordo superior esquerdo.
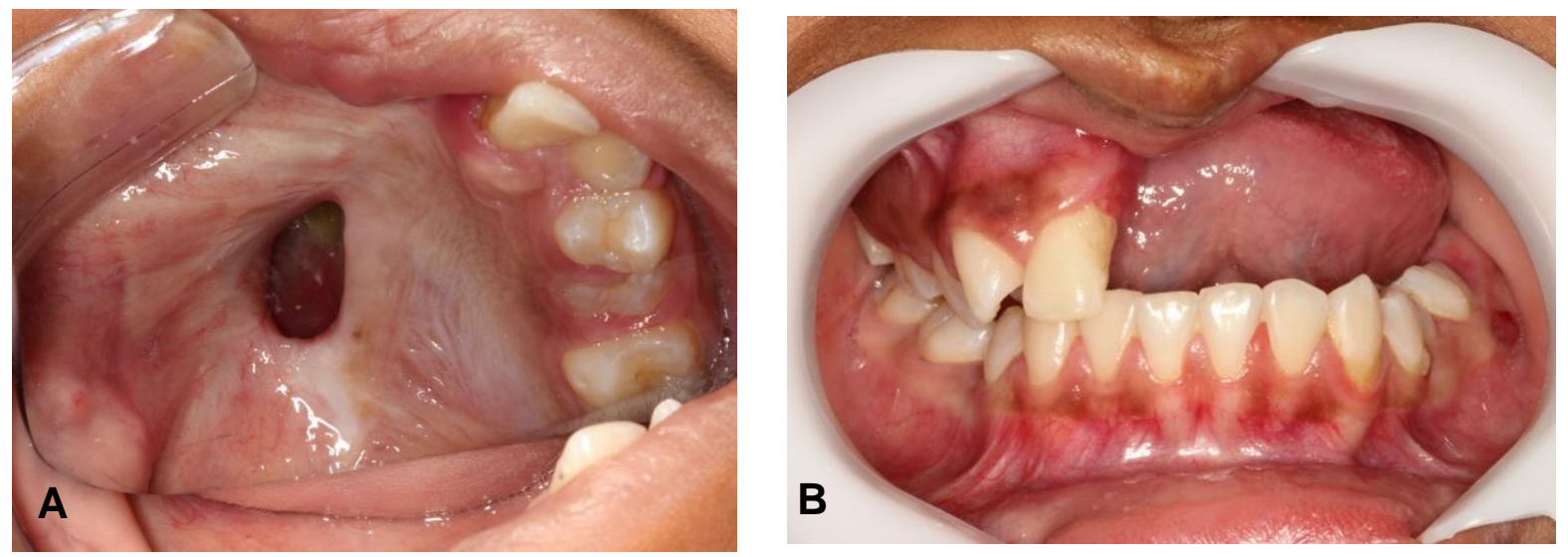

Fonte: Autores.

Na Figura 2 observa-se o aspecto inicial da cavidade bucal com a presença da comunicação bucosinusal na região hemimaxilar esquerda, a ausência do rebordo alveolar esquerdo assim como de alguns elementos dentários e a paciente na posição de relação cêntrica.

O tratamento foi iniciado pela moldagem anatômica das arcadas superior e inferior com alginato. Na moldagem da maxila a área da comunicação bucosinusal foi protegida com gaze para que não houvesse extravasamento de material para a cavidade e o alginato foi manipulado em consistência mais densa para melhor reprodução da estrutura maxilar perdida (Figura 3). Com os modelos de estudo obtidos foram realizados o delineamento e planejamento do caso, para determinação do eixo de inserção, confecção das coroas guias, área de retenção e desenho da armação metálica. 
Figura 3: Moldes das arcadas superior e inferior.

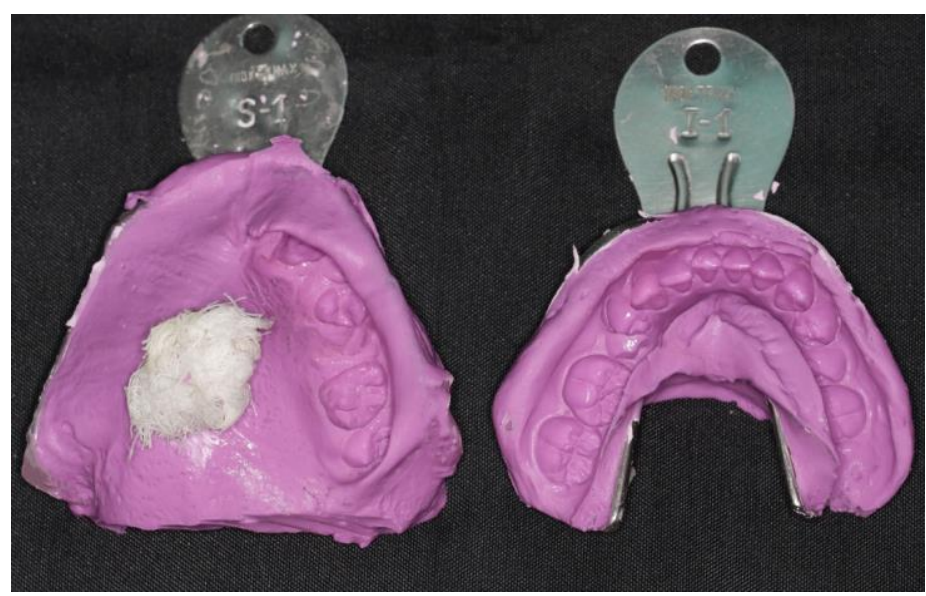

Fonte: Autores.

Na Figura 3 percebe-se os detalhes dos moldes superior e inferior, sendo que no superior há presença da gaze que foi inserida na comunicação bucosinusal para protegê-la e a ausência de rebordo alveolar do lado esquerdo.

Em seguida foram executados os preparos dos nichos nos elementos 12 (distal) e 13 (mesial) em que foram idealizados apoios de cíngulos e foram confeccionados nichos na face oclusal dos elementos 16 (distal) e 17 (mesial) em que planejou-se grampos circunferenciais geminados. Foi realizada a moldagem funcional da arcada superior com alginato (Figura 4), para obtenção do modelo de trabalho sobre o qual foi confeccionada a armação metálica (Figura 5). Após prova da armação metálica na boca, foi confeccionado o pano de cera sobre a sela da armação metálica. Foi realizado o registro do arco facial e a montagem modelo superior no articulador semiajustável (ASA). O modelo inferior foi montado em ASA após os registros intermaxilares. Em seguida, foi realizada a seleção dos dentes artificiais levando em conta a cor e o tamanho dos dentes remanescentes, idade, sexo, cor da pele, formato do rosto e as linhas de referências demarcadas no plano de cera.

Figura 4: Molde superior após confecção dos nichos.

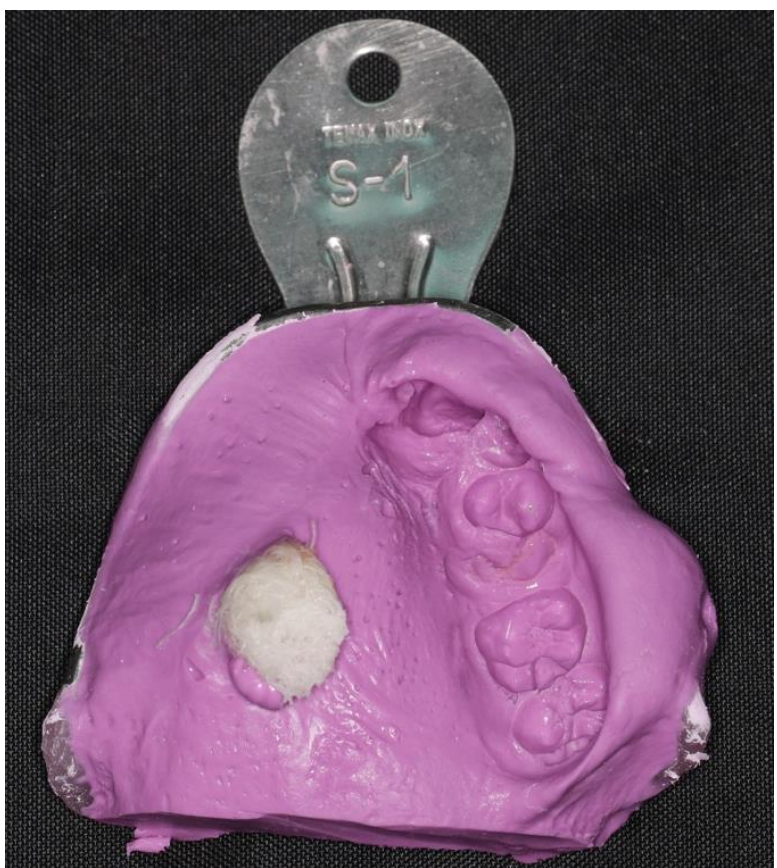

Fonte: Autores. 
A Figura 4 mostra o molde confeccionado com hidrocolóide irreversível após preparo dos nichos nos dentes de apoio, verifica-se também a gaze que protegeu a comunicação bucosinusal e a ausência de rebordo do lado esquerdo.

Figura 5: Modelo de trabalho superior e armação metálica. A) Modelo em gesso. B) Armação metálica.
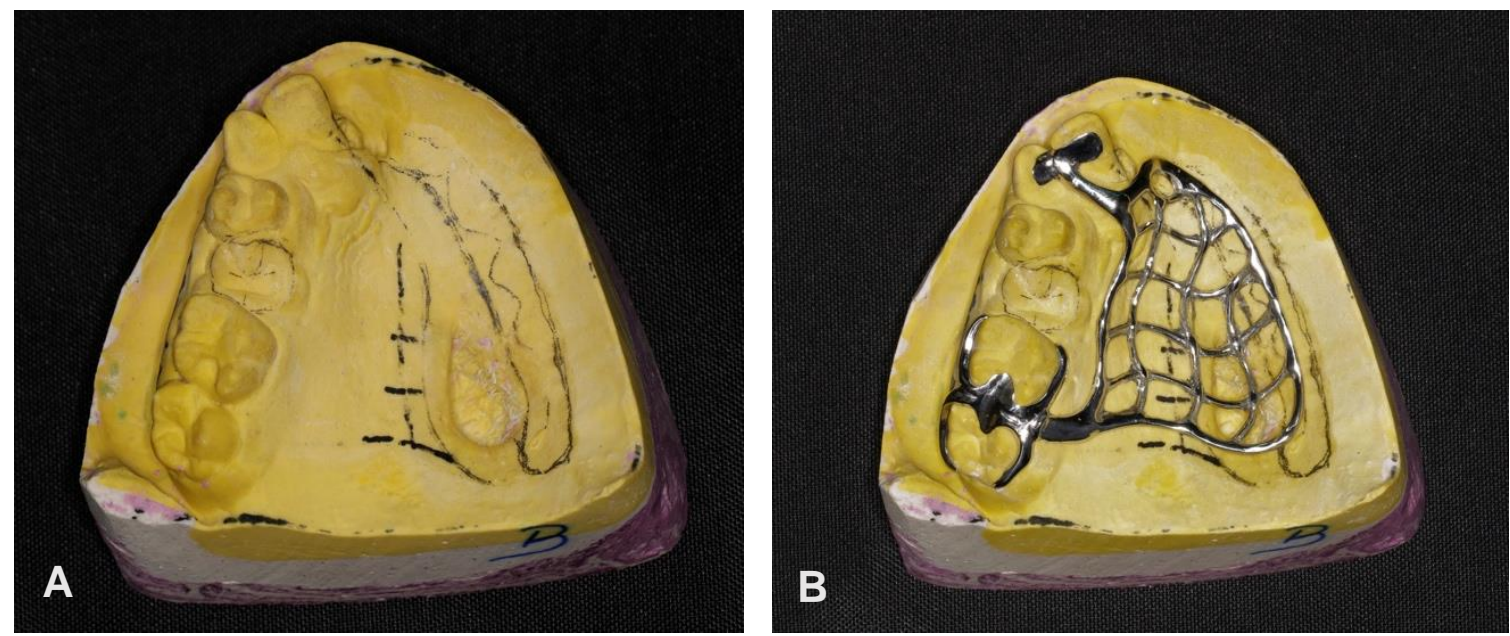

Fonte: Autores

A Figura 5 mostra o modelo de trabalho obtido após a moldagem funcional e a armação metálica da prótese parcial removível confeccionada sobre este modelo. Não foi utilizado grampos nos pré-molares porque o $2^{\circ}$ premolar não estava completamente erupcionado.

Os dentes artificiais formaram montados e foi realizada a prova estética e funcional na cavidade bucal, onde foram verificados suporte de lábio, altura incisal, corredor bucal, linha mediana, linha alta do sorriso e as características dos dentes artificiais como cor, tamanho e forma. Além disso, foram executados ajustes oclusais, testes fonéticos e testes de retenção (Figura 6 e 7). Após a análise dos parâmetros, que estavam adequados e satisfatórios, foi realizada a moldagem funcional de boca fechada com silicone de consistência leve (Figura 8). A prótese foi enviada para os procedimentos laboratoriais de acrilização, acabamento e polimento (Figura 9).

Figura 6: Dentes artificiais montados em cera.

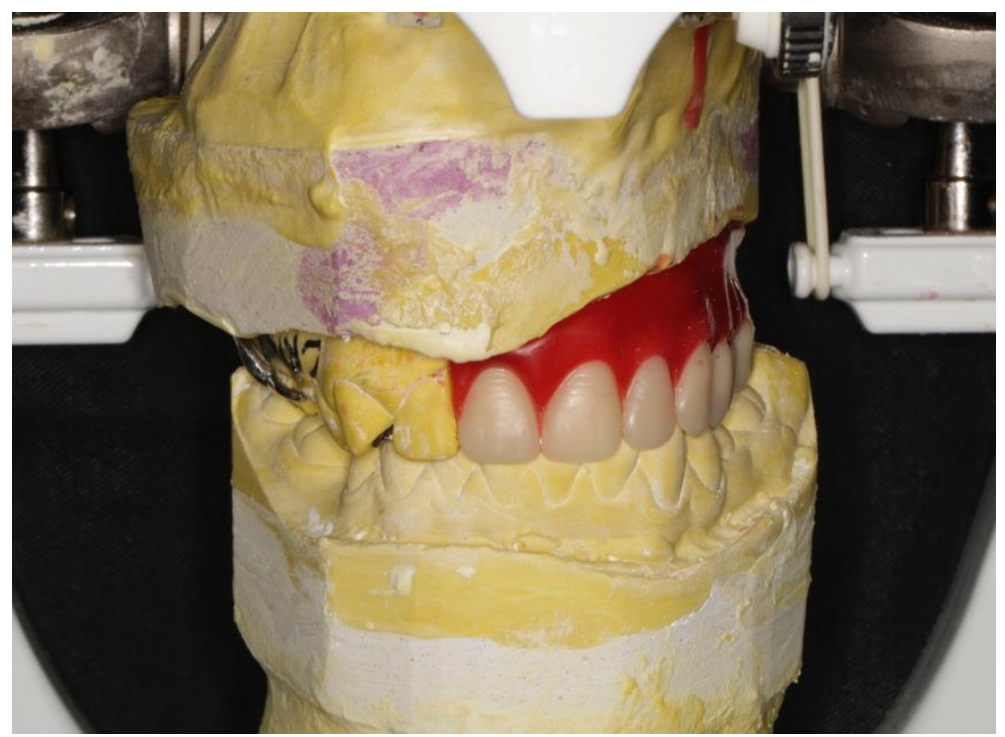

Fonte: Autores. 
Na Figura 6 tem-se uma vista frontal dos modelos montados em ASA com os dentes artificiais montados em cera, aonde pode-se avaliar a oclusão e o alinhamento dos dentes superiores.

Figura 7: Prova estética e funcional dos dentes artificiais.

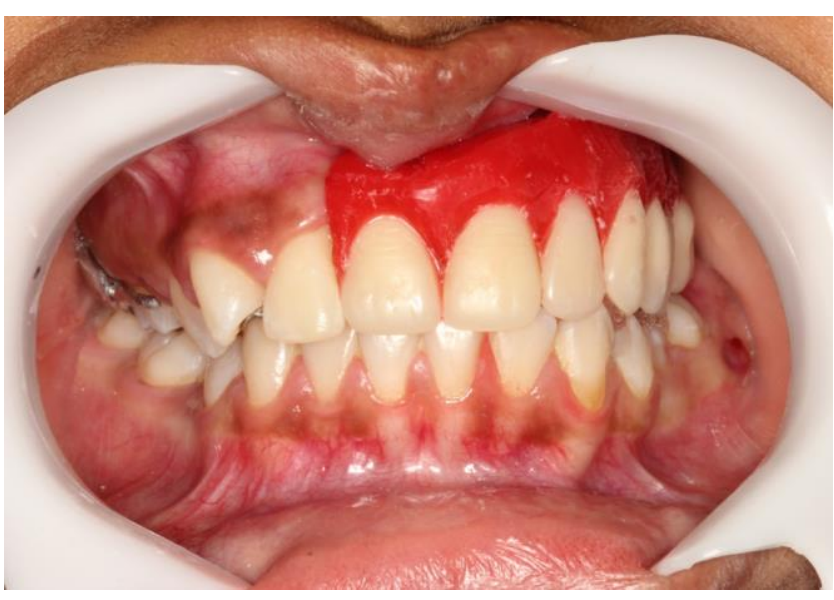

Fonte: Autores.

Na Figura 7 é possível avaliar a prova estética e funcional dos dentes e constatar a correta oclusão e adequada cor e tamanho dos dentes o que permitiu um sorriso satisfatório e uma melhora na fonética.

Figura 8: Após moldagem de boca fechada. A) Vista superior. B) Vista posterior.
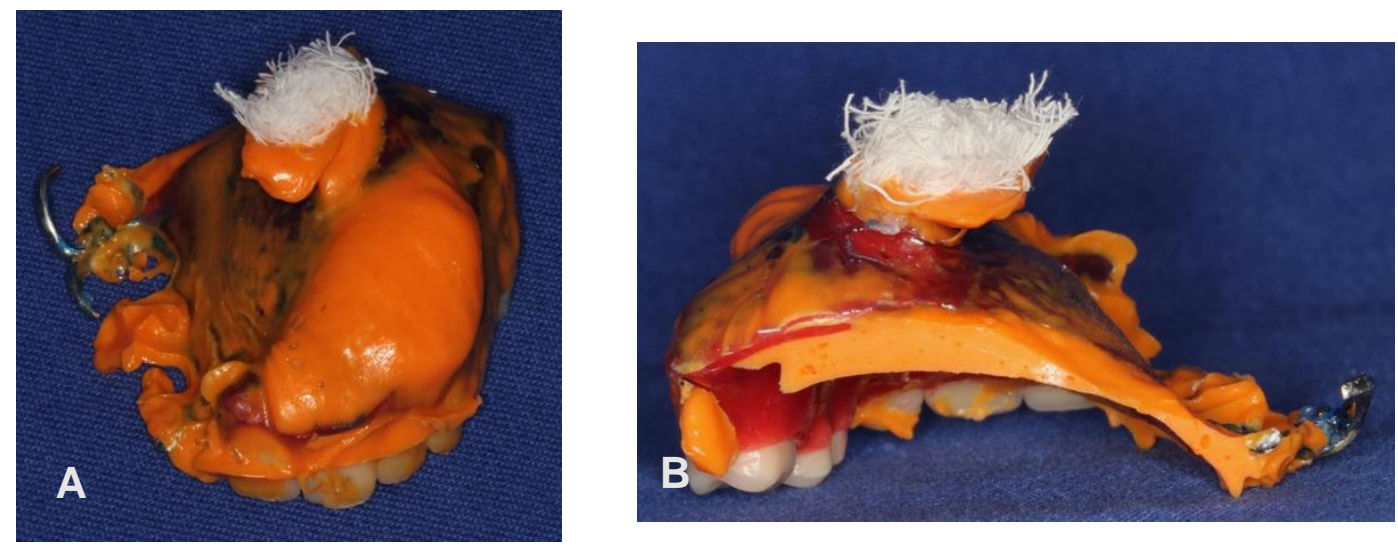

Fonte: Autores.

Na Figura 8 observa-se a vista superior e posterior da moldagem de boca fechada realizada com silicone de condensação na consistência leve e percebe-se com maior detalhe toda extensão da área que será abrangida pela prótese. 
Figura 9: Prótese obturadora superior concluída. A) Vista superior. B) Vista frontal.
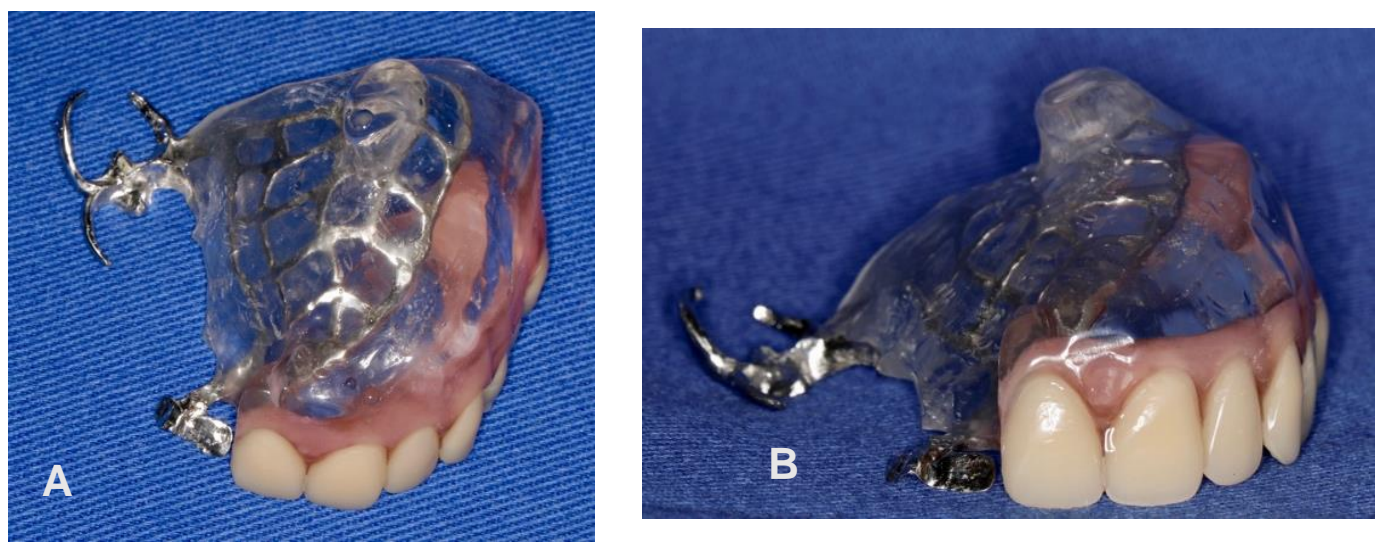

Fonte: Autores.

Na Figura 9 percebe-se uma vista superior e frontal da prótese obturadora finalizada com a porção metálica palatina envolvida com acrílico incolor para permitir o vedamento da comunicação bucosinusal.

Em seguida a prótese parcial removível superior com obturador palatino foi instalada e os testes fonéticos, testes de retenção e estabilidade, contatos oclusais e princípios estéticos foram novamente avaliados (Figura 10 e 11). A paciente recebeu informações sobre a inserção, remoção e higienização da prótese. A primeira proservação foi realizada 48 horas após a instalação, a paciente mostrou-se satisfeita com a estética e retenção e relatou melhora na deglutição, mastigação e fonação. Na segunda proservação realizada sete dias após a instalação, a paciente relatou que a prótese estava bem adaptada e que não lhe causava nenhum incomodo. Desta forma, foi orientada a retornar após um ano.

Figura 10: Prótese superior instalada. A) Vista intraoral. B) Sorriso final.
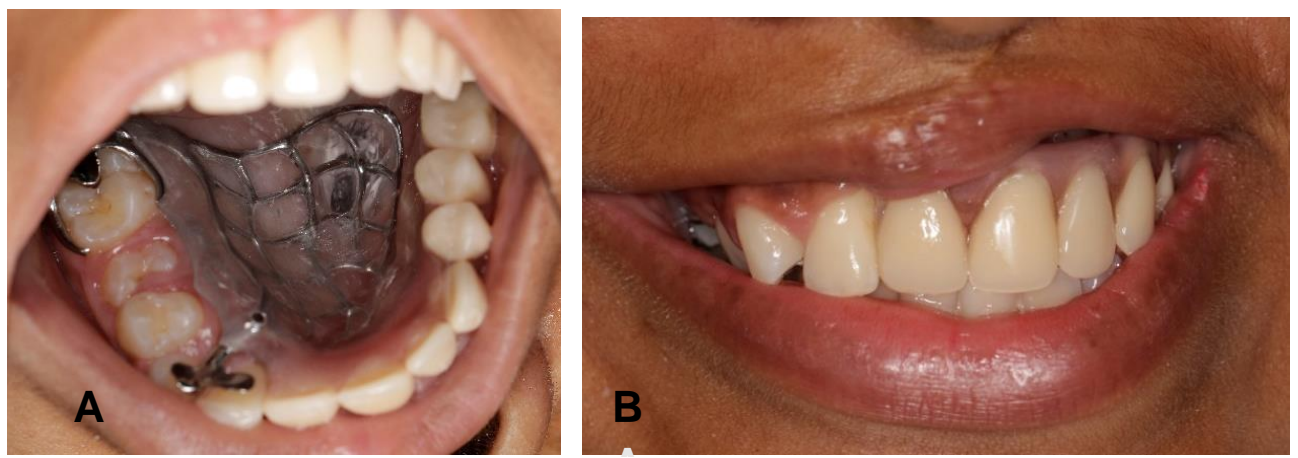

Fonte: Autores.

A Figura 10 mostra uma vista intraoral oclusal da prótese instalada aonde verifica-se a boa adaptação da prótese obturadora e o selamento da comunicação bucosinusal. O sorriso final ficou bastante satisfatório tanto na cor dos dentes artificiais quanto no alinhamento. 
Figura 11: Aspecto clínico final. A) Frontal. B) Perfil direito. C) Perfil esquerdo.
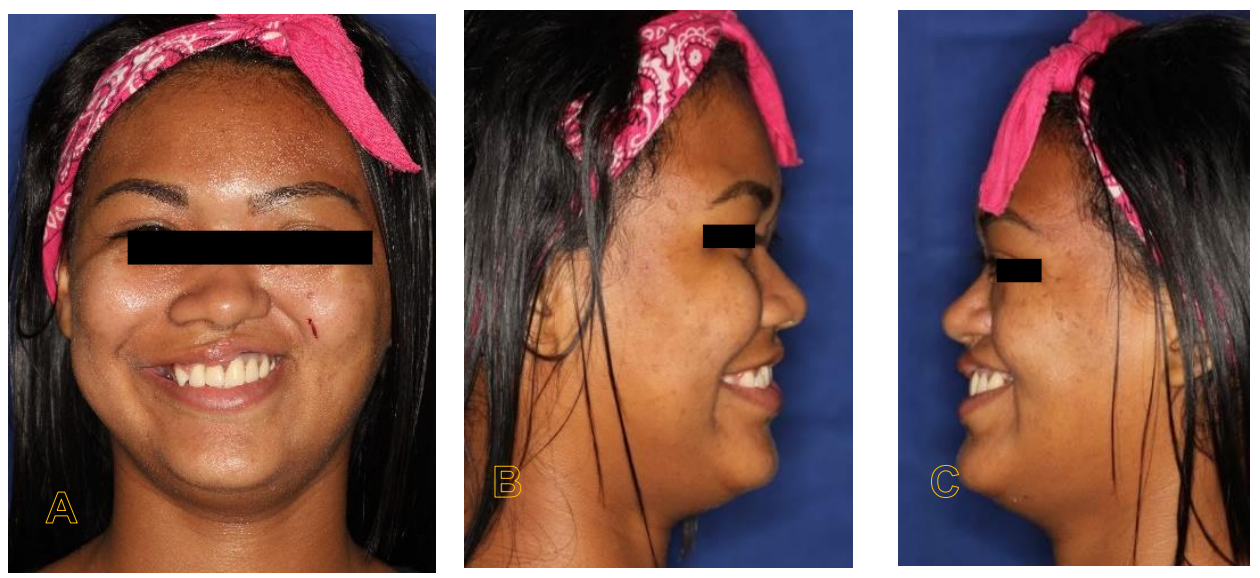

Fonte: Autores.

Na Figura 11 observa-se o aspecto facial da paciente após a instalação da prótese, na vista frontal percebe-se uma melhora significante no sorriso o que trouxe grande satisfação para paciente. Também é possível observar uma maior harmonia entre os lados direito e esquerdo.

\section{Resultados e Discussão}

O Osteoma é uma neoplasia benigna que afeta os ossos da região maxilofacial, não possui predileção por gênero, ocorre geralmente entre os 30 e 50 anos. Apresenta-se radiograficamente como um tecido ósseo esclerótico radiopaco bem delimitado, podendo ter relação com a síndrome de Gadner (Caubi et al., 2013). O tumor tem como regiões anatômicas preferenciais a maxila, mandíbula e os seios paranasais (Neville et al., 2016). As lesões ósseas podem se originar de anomalias do desenvolvimento, neoplasias verdadeiras ou lesões reacionais associadas a eventos traumáticos ou infecciosos (Carrera et al., 2009; Durão et al., 2012). O tratamento do osteoma constitui-se na excisão cirúrgica da lesão, que quando localizada no palato duro é denominada maxilectomia parcial ou total (Boros et al., 2011). O caso clínico apresentado diverge da literatura, pois acometeu a paciente com onze anos. Em contrapartida, assemelhando-se aos estudos, a região maxilar também foi a mais prevalente no relato, a paciente foi diagnosticada com osteoma e submetida a maxilectomia parcial.

A reabilitação protética é a prática mais habitual de tratamento não traumático, objetivando a substituição dos tecidos que foram perdidos, melhorando a deglutição, fonação e mastigação (Dalkiz et al., 2018). A remoção cirúrgica implica diretamente no grau de estabilidade, suporte e retenção. O caso clínico abordado ratifica os estudos de Ali et al., (2018) afirmando que o número de elementos dentários remanescentes, a atividade muscular, a extensão e profundidade das deformidades ósseas, a mucosa ao redor do defeito, a altura da crista do rebordo e o peso das próteses estão intimamente ligadas a ausência da estabilidade, suporte e retenção (Aramany, 1978; Aramany, 1978).

Os defeitos maxilares apresentam diversos sistemas classificatórios, sendo a classificação de Aramany (1978) a mais comum. Possuindo seis tipos de deformações ósseas, na classe I a dentição e o ósseo alveolar removidos não atravessam a linha média, sendo o hemiarco contralateral preservado. Já a classe II evidencia que pré-maxilar do arco da ressecção cirúrgica é preservado, a classe III mostra que toda a dentição é preservada e apenas o palato é envolvido no defeito, na classe IV a dentição e osso removidos cruzam a linha média e só os dentes posteriores são preservados. Na classe V a ressecção cirúrgica ocorre na porção maxilar posterior. Por fim, a classe VI envolve o pré-maxilar, preservando os dentes posteriores (Aramany, 1978; Aramany M, 1978; Pereira, 2014; Corsalini et al., 2021). 
Segundo Ali et al., (2018) o grau de maxilectomia (parcial ou total) e a classificação de Aramany estão associados ao tipo de retenção alcançada pelo obturador protético (Ali et al., 2018). Sendo a classe IV a mais favorável no sucesso da reabilitação oral, diferente das demais classes que apresentam grau de retenção e estabilidade reduzido. (Corsalini et al., 2021). No caso clínico relatado, a paciente era edentada parcial do lado esquerdo, possuía uma pequena cavidade bucosinusal, sendo classificada na classe I de Aramany, com isso foi observado dificuldade elevada na obtenção biomecânica, corroborando com os estudos antecessores. Devido as particularidades do caso optou-se pela confecção de uma prótese parcial removível com obturador palatino.

A prótese parcial removível deve apresentar os conectores maiores rígidos, possuindo íntimo contato com o palato, promovendo maior suporte. Os apoios devem transmitir as forças oclusais no sentido do longo eixo do dente e os planos-guias precisam garantir a manutenção da estabilidade, suporte e retenção (Singh et al., 2013; Carreiro et al., 2008; McCraken, 2012).

A extensão do defeito maxilar influencia na biomecânica das próteses obturadoras, pois gera forças de alavanca e linhas de fulcro, aumentando a exigência durante os esforços mastigatórios (Miyashita et al., 2011). A altura, o contorno do rebordo, a profundidade dos sulcos, o número, posição e o estado periodontal dos dentes remanescentes of erecem uma melhor retenção (Singh et al., 2013; Pereira, 2014). Os retentores circunferenciais são indicados quando só existem dentes restantes em um lado do rebordo (Pereira, 2014; Keyf, 2001). Conforme a literatura relata, no caso foi observado uma maior força de alavanca devido a paciente apresentar somente uma hemiarcada, a boa saúde do periodonto de suporte e sustentação contribuiu para um melhor assentamento do obturador palatino, sendo o uso de grampos circunferenciais geminados de grande valia para alcance da retenção e estabilidade protética do relato.

Outros meios de retenção estão baseados na reabilitação por implantes dentários osteointregados, que podem possuir encaixes do tipo O'ring, barra clipe, sistema ERA e magnetos (Kusterer et al., 2012). Os sistemas O’ring é composto por duas partes, uma parafusada ao implante e outra fixada na prótese. Esse sistema necessita de correto paralelismo entre os implantes, deve possuir no máximo 10 graus de divergência, é indicado para sobredentaduras ligadas com implantes independentes. O encaixe do tipo barra-clipe promove a união dos implantes à prótese por intermédio de clipes plásticos ou metálicos aderidos a estrutura protética, possui como indicação os casos de restauração mandibulares. Já o Sistema Era possui dois componentes de encaixes, a porção fêmea, presa ao implante e a porção macho retida na prótese do paciente, é utilizado em overdenture dentossuportada e prótese parciais removíveis. Por fim, o sistema magnético é caracterizado pela utilização de pequenos imãs, sendo indicado para áreas de baixa qualidade óssea (Novaes et al., 2008; Aquino et al., 2005).

O uso dos implantes dentários nas próteses obturadoras palatinas restringisse-se a área do defeito maxilar e estrutura óssea remanescente, os implantes vizinhos a comunicação bucosinusal apresentam maior predisposição a reabsorção óssea (Pereira, 2014; Monae et al., 2009). A condição bucal e sistêmica tem grande influência na longevidade dos implantes dentários, pois a união do implante ao osso alveolar, depende de fatores como bom suporte periodontal e adequada irrigação sanguínea (Gomes et al., 2022). As próteses implanto-suportadas possuem retenção superior as próteses parciais removíveis e as estritamente mucosa-suportadas. No entanto, possuem custo elevado, maior rejeição pelo paciente e risco acentuado de osteoradionecrose em pacientes submetidos a radioterapia (Soares et al., 2008). A escolha do sistema de retenção ideal é fundamental para sucesso dos obturadores protéticos, deve-se sempre buscar alternativas retentivas que melhorem a longevidade das próteses (Parize et al., 2021). Sendo o uso de implantes inviável ao caso, devido a pouca idade da paciente e alto custo dos implantes, além da rejeição da mesma por novos procedimentos cirúrgicos, no caso clínico foi confeccionada uma prótese parcial removível com obturador palatino.

\section{Considerações Finais}

A prótese bucomaxilofacial ainda possui grandes desafios em sua confecção, sendo o principal deles relacionado aos 
tipos de retenção. No caso apresentado foi escolhido a retenção por meio de prótese parcial removível, para isso é necessário analisar o número, a posição e o estado periodontal dos dentes remanescentes. Bem como realizar um adequado planejamento levando em consideração as particularidades dos defeitos maxilares. Uma alternativa seria a retenção por meio de implantes dentários, no entanto esta possibilidade foi rechaçada pela pouca idade da paciente, além de apresentar ônus elevado e mais intervenções cirúrgicas.

O obturador palatino fixado com prótese parcial removível, apesar de não ser o meio mais retentivo, mostrou-se eficaz esteticamente e funcionalmente, restabelecendo as funções mastigatórias, a fonação, deglutição e devolução ao convívio social. Além de possuir baixo custo e rapidez na reabilitação bucal da paciente.

Faz-se necessário mais estudos e publicações sobre os sistemas de retenção para próteses obturadoras palatinas, pois este tema é de grande relevância tendo em vista o grande número de pacientes que procuram este tipo de prótese após terem sido submetidos a cirurgias oncológicas.

\section{Referências}

Ali, M. M., Khalifa, N., \& Alhajj, M. N. (2018). Quality of life and problems associated with obturators of patients with maxillectomies. Head \& Face Medicine, 14 (1), 2.

Aquino, E. B., Alves, B. P., \& Filho J. N. A. (2005). Sistemas de Encaixes Utilizados em Overdentures Implantossuportadas. PCL, 7(36),159-65.

Aramany, M. A. (1978). Basic principles of obturator design for partially edentulous patients. Part I: Classification. J. Prosthet. Dent, 40, 554-557. doi: 10.1016/0022-3913(78)90092-6.

Aramany, M. A. (1978). Basic principles of obturador design for partially edentulous patient. Part II: design principles. J. Prosthet. Dent, 40(5):656-662

Boros, L. F., Carneiro, M. I. S., Boros, L. H., \& Boros P. A. S. (2011). Osteoma compacto central de mandíbula: relato de caso clínico. Odontol. Clín-Cient , $10(1)$.

Caubi, A. F., Moura, R. Q., Borba, P. M., Costa, D. F. N., \& Bispo, L. M. M. (2013) Osteoma em mandíbula: quando tratá-lo cirurgicamente. Rev. Cir. Traumatol. Buco-Maxilo-Fac., Camaragibe. 13 (1), 53-58.

Carrera M., Pereira Jr F. B., Santos, J. N., \& Vasconcelos, R. J. H. (2009). Osteoma periférico en lamandíbula: Presentación de caso. Acta odontol. venez, 47(2), 376-382.

Carreiro, A. D. P., Bezerra, C. F. R., Amaral, B. A, Piuvezam, G., \& Seabra, E. D. (2008). Aspectos Biomecânicos das Próteses Parciais Removíveis e o Periodonto de Dentes Suporte. R. Periodontia. 18(01),105-113.

Corsalini, M., Barile, G., Catapano, S., Ciocia, A., Siciliani, R., Di Venere D., \& Capodiferro, S. (2021). Obturator Prosthesis Rehabilitation after Maxillectomy: Functional and Aesthetical Analysis in 25 Patients. Int J Environ Res Public Health. 18 (23),12524.

Dalkiz, M., \& Dalkiz, A. S. (2018). The Effect of Immediate Obturator Reconstruction after Radical Maxillary Resections on Speech and other Functions. Dent J (Basel). 6 (3), 22.

Durão, A. R., Chilvarquer, I., Hayek, J. E., \& Provenzanom, \& Kendall, M. R. (2012). Osteoma of the zygomatic arch and mandible: Report of two cases. Rev Port EstomatolMed Dent Cir Maxilofac, 53(2),103-107.

Dings, J. P. J., Merkx, M. A., Maclennan-Naphausen, M. T. P., Pascal van de Pol, Maal, T. J. J., \& Meijer, G. J. (2018). Maxillofacial prosthetic rehabilitation: A survey on the quality of life. The Journal of Prosthetic Dentistry, 120(5), 780-786.

Goiato, M. C., Pesqueira, A. A., Ramos da Silva, C., Gennari Filho, H., \& Micheline Dos Santos, D. (2009). Patient satisfaction with maxillofacial prosthesis. Literature review. Journal of plastic, reconstructive \& aesthetic surgery: JPRAS, 62(2), 175-180.

Gomes, A. V. S. F. ., Vieira, M. C. de S. ., Flor, L. C. de S. ., Trinta, L. B. ., Sousa , A. C. A. ., Santos , A. C. C. ., Coelho , Y. B. S. ., Lindoso, E. T. C. ., Mousinho, L. E. C. ., Campos, S. M. ., Almeida, J. C. ., Freitas, C. V. S. de ., Demétrio , M. S. ., Bazán, J. M. N. ., \& Agostinho, C. N. L. F. (2022). Influence of bariatric surgery on dental implant treatment. Research, Society and Development, 11(1), e48411125090. https://doi.org/10.33448/rsd-v11i1.25090

Keyf, F. (2001). Obturator Prostheses for hemimaxillectomy patients. Journal of Oral Rehabilitation, 28 (9), $821-829$.

Kusterer, L. E. F. L., Paraguassú, G. M., Silva, V. S. M., \& Sarmento, V. A. (2012). Reabilitação com obturador maxilar após cirurgia oncológica: relato de casos. Rev cir traumatol buco-maxilo-fac. (4),9-16.

Miyashita, E. R., Mattos, B. S. C., \& Marafon, P. G. (2011). Sistemas de Retenção em Prótese Obturadora Cirúrgica Para Pacientes Maxilectomizados. Pesquisa Brasileira em Odontopediatria e Clínica Integrada. 11(2), 263-268.

McCracken, C. A. B.; \& Brown, D. T. (2012). Prótese Parcial Removível (12. ed.). Rio de Janeiro, RJ: Editora Elsevier.

Monae, M., \& Shaker, K. (2009). Use of osseointegrated implants to retain obturators of edentulous patients, CDJ, 25 (1),1-8. 
Research, Society and Development, v. 11, n. 3, e11611326468, 2022

(CC BY 4.0) | ISSN 2525-3409 | DOI: http://dx.doi.org/10.33448/rsd-v11i3.26468

Neville, B. W., Damm, D. D, Allen, C. M, \& Bouquot, J. E. (2016). Patologia oral e maxilofacial. (4ª ed.). Rio de Janeiro: Elsevier.

Novaes, L. C. G. F., \& Seixas, Z. A. (2008). Prótese total sobre implante: técnicas contemporâneas e satisfação do paciente. Int J Dent. 7(1), 50-62.

Parize, G., Pestana T. I., Cardoso, R. F., Kim, Y. J., \& Pallos, D. (2021). Prótese sobre implante dentário parafusada ou cimentada?. Research, Society and Development, 10 (15). https://doi.org/10.33448/rsd-v10i15.23112

Pereira, A.S. et al. (2018). Metodologia da pesquisa científica. [e book]. Santa Maria.Ed.UAB/NTE/UFSM.

Pereira, A. M. L. (2014). Reabilitação Oral em Pacientes Maxilectomizados. Porto: Universidade Fernando Pessoa- Faculdade de Ciências da Saúde.

Singh, M., Bhushan, A., Kumar, N., \& Chand, S. (2013) Obturator prosthesis for hemimaxillectomy patients. National Journal of Maxilofacial Surgery 4(1), 117-120.

Soares, A., Dib, L., \& Piras de Oliveira, J. (2008). Reabilitação de pacientes oncológicos maxilectomizados: uma abordagem multidisciplinar. Revista ImplantNews, 5 (1), 21-26. 